\title{
La rivista Crocevia. Esperienze di una (breve) direzione
}

Daniele Comberiati

\section{(2) OpenEdition \\ 1 Journals}

Edizione digitale

URL: http://journals.openedition.org/esp/3271

DOI: 10.4000/esp.3271

ISSN: 2532-0319

Editore

Centre d'Information sur l'Éducation Bilingue et Plurilingue

\section{Edizione cartacea}

Data di pubblicazione: 1 décembre 2018

Paginazione: 89-96

ISSN: 1127-266X

\section{Notizia bibliografica digitale}

Daniele Comberiati, « La rivista Crocevia. Esperienze di una (breve) direzione », Éducation et sociétés plurilingues [Online], 45 | 2018, Messo online il 01 décembre 2020, consultato il 04 décembre 2020. URL : http://journals.openedition.org/esp/3271 ; DOI : https://doi.org/10.4000/esp.3271 


\section{LA RIVISTA CROCEVIA. ESPERIENZE DI UNA (BREVE) DIREZIONE}

\section{Daniele Comberiati}

À partir de son expérience en tant que directeur de la revue Crocevia, publiée par l'éditeur Besa, l'auteur réfléchit sur l'évolution des études concernant la «littérature italienne de la migration» et surtout sur la notion de «littérature nationales. À l'heure actuelle, où les phénomènes migratoires demeurent un des éléments les plus importants du contexte contemporain, la coexistence, dans le même lieu, des personnes ayant des langues et cultures différentes devrait nous pousser à concevoir différemment l'idée de "littérature nationale» et à reconsidérer les liens entre langue et littérature.

Mots clés: revue Crocevia, littérature italienne de la migration, littérature nationale, langue et littérature

Taking off from his own experience as director of Crocevia, journal published by Besa, the author reflects upon the evolution of studies that bear on the "Italian literature of migrations», and especially on the notion of a «national literatures. At present, when migrations are one of the most important factors in our contemporary contexts, the coexistence, in the same place and time, of people of different languages and cultures should incite us to take a different view of a snational literatures and to reconsider the links between language and literature.

Key-words: journal Crocevia, Italian literature of migrations, national literature, language and literature

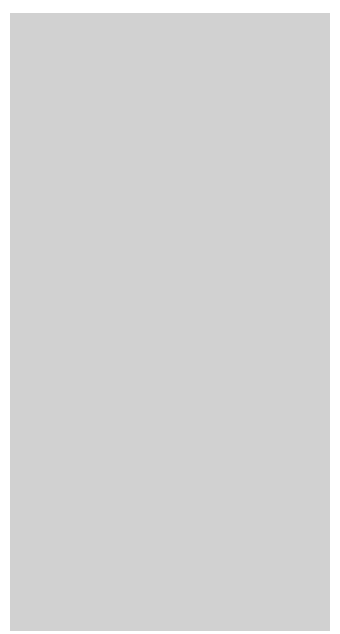

Q

uando ho iniziato ad occuparmi di "letteratura italiana della migrazione", ovvero delle produzioni in lingua italiana degli scrittori migranti, era il 2004 e non molte erano le opere scientifiche sul tema. Vi erano i lavori pionieristici di Armando Gnisci (Gnisci 1998), alcuni validi contributi teorici oltreoceano di Graziella Parati (Parati 1999) e altri studi ugualmente derivanti dal Dipartimento di Letterature Comparate dell'Università La Sapienza di Roma, nel quale Gnisci insegnava (Sinopoli 2001). Avevo iniziato un dottorato all'Université Libre de Bruxelles, con la supervisione di Claudio Gigante, e la questione linguistica appariva fin dall'inizio particolarmente problematica: lavorando in un paese di emigrazione italiana storica, la tematica della mia tesi incrociava altri e già più studiati percorsi. Chi erano infatti questi "scrittori italiani migranti"? Potevamo annoverare al loro interno anche gli scrittori italiani emigrati all' estero (o figli o nipoti di italiani emigrati all'estero), oppure il percorso migratorio (e dunque di conseguenza quello linguistico) andava inteso in un solo 


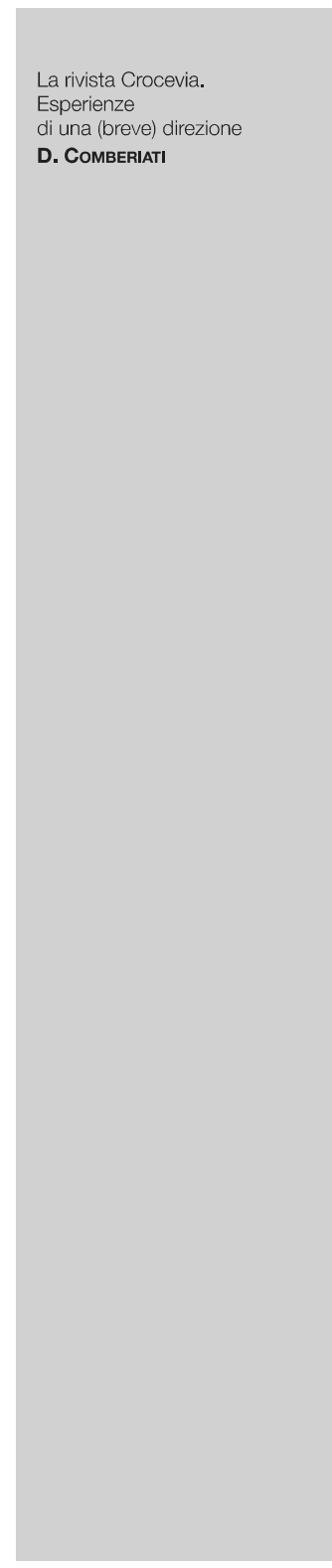

senso e verso una sola direzione? Ricordo bene una interessante discussione con il jury de thèse, all'indomani della presentazione del mio progetto di ricerca: il titolo iniziale del mio lavoro era infatti Ecrire dans la langue de l'autre: la litterature des migrants en Itakie (1989-2007) [Scrivere nella lingua dell'altro: la letteratura dei migranti in Italia (1989-2007)], ma la commissione aveva dei dubbi proprio su quel "migrants": a chi si riferiva? Agli immigrati o agli emigranti? Non era forse il caso di specificarlo, visto che poi nella tesi parlavo quasi esclusivamente di scrittori stranieri giunti in Italia a partire dalle ondate migratorie degli anni Ottanta? Il titolo finale, da cui il libro pubblicato nel 2010, porta il segno di tali discussioni: Scrivere nella lingua dell'allro: la letteratura degli immigrati in Italia (1989-2007) accetta $\mathrm{e}$ comprende i dubbi posti allora dalla commissione (Comberiati 2010), c riporta la questione della letteratura della migrazione ad un contesto linguistico. La lingua degli scrittori migranti è una lingua di "resistenza" (come nel caso di scrittori emigrati che continuano ad usare l'italiano in un contesto allofono) o di "(ri)appropriazione" (come nel caso di scrittori stranieri che, in un contesto italofono, si "appropriano" di una lingua altra)? Il titolo fa riferimento anche al lavoro di Assia Djebar, Ces voix qui m'assiègent, saggio-pamphlet che dice diverse cose interessanti sul rapporto fra scrittore (anzi scrittrice) migrante e lingua (Djebar 1999). Per Assia Djebar, scrittrice algerina, non è il fatto di usare il francese che comporta l'impiego della lingua "altra", né lo sarebbe stato l'uso della lingua araba: in un contesto storico-politico e sociale in cui le donne hanno avuto poche possibilità di far sentire la propria voce, prendere la parola attraverso la scrittura, con qualsiasi lingua, è già di per sé un atto politico e civile rivoluzionario. La "lingua dell'altro" è nella presa di parola laddove il soggetto parlante era considerato non abilitato a parlare, mentre secondaria è la scelta della lingua. È una questione che è stata molto importante per gli scrittori migranti di espressione italiana nei primi anni Novanta: cimentarsi con una lingua nuova non sempre ha comportato un abbandono della lingua originaria, e sono diversi i casi di scrittori che hanno continuato a scrivere "anche" nella loro lingua d'origine, non distinguendo affatto, nella loro produzione intellettuale, fra "prima" e "seconda" lingua, ma utilizzando un idioma specifico per determinate tematiche.

Il concetto stesso di italofonia, al tempo, era già messo in discussione. Letteralmente "italofono" indica il parlante la lingua italiana, senza distinzioni di origine o nazionalità. Il problema era però che, negli studi letterari, non era la lingua orale italiana che veniva presa in considerazione, ma le pratiche letterarie scritte in italiano. Per tale ragione alcuni critici, Franca Sinopoli su tutti, avevano proposto di utilizzare piuttosto la nozione di italografia (meglio ancora nella sua declinazione plurale: italografie) per indicare le scritture in lingua italiana, che a questo punto 


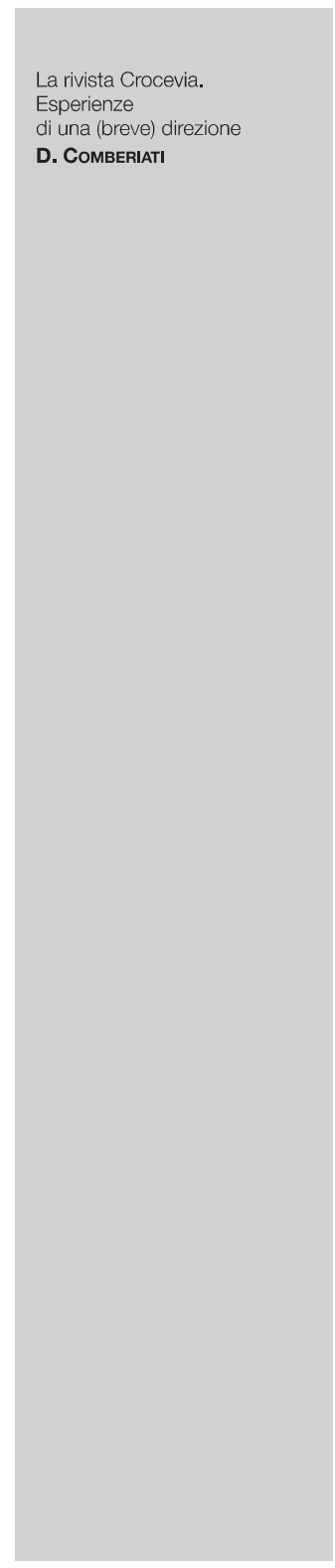

potevano riguardare indistintamente le opere di scrittori immigrati giunti in Italia o le pubblicazioni degli italiani all'estero (Sinopoli 2006: 185).

A questa problematica se ne legava immediatamente un'altra, di natura teorica: se la scrittura in italiano superava i confini storico-politici della nazione, aveva ancora senso parlare di letteratura nazionale e concepire gli studi e i percorsi letterari basandosi su tale concetto? È ovviamente una domanda che esula dal mero studio sulla letteratura italiana della migrazione e che mette in gioco tutta una serie di abitudini culturali attraverso le quali siamo soliti analizzare e studiare le storie letterarie. Un articolo del 2006, scritto dall'arabista Estella Carpi, riprendeva il concetto da un punto di vista opposto (Carpi 2006): gli scrittori migranti di origine araba sono da considerare afferenti alla letteratura italiana solo perché utilizzano l'italiano per la loro produzione? L'atto linguistico è dunque sufficiente per appartenere ad una tradizione letteraria (per la quale la lingua è importante, ma non è l'unico parametro che può essere preso in considerazione) oppure dovrebbero essere altri gli elementi di appartenenza, quali la conoscenza e la relazione con gli autori della letteratura in questione, la partecipazione ad un immaginario comune o infine la condivisione di stili, tematiche e tipologie espressive? La tesi dell'autrice, ovviamente, verteva su questo secondo punto: per lei, provocatoriamente, gli autori italofoni (o italografi) di origine araba dovevano essere considerati come facenti parte della letteratura araba e non di quella italiana, "trascurando" in un certo senso la scelta linguistica in favore di altri parametri. Personalmente mi trovavo (e mi trovo) in disaccordo con le sue posizioni, ma mi interessava particolarmente la porosità della sua affermazione e gli squarci che apriva all'interno di una visione della letteratura sclerotizzata e considerata "naturale", dunque difficile da modificare.

Un recente libro, edito da Quodlibet, riprende la questione da un punto di vista ulteriore (Baldini, Biagi, De Lucia, Fantappiè, Sisto 2018). Catalogando e analizzando le traduzioni in italiano dal tedesco in un preciso arco di tempo, gli autori e le autrici del volume si sono resi conto dell'importanza di questi testi nell'evoluzione della letteratura italiana coeva. Sono opere che hanno dialogato con quelle degli scrittori italiani del tempo, e, forti della loro legittimità culturale perché provenienti da un paese e da una lingua considerata all'epoca come portatrice di una tradizione e di un impatto letterario maggiori, hanno contribuito ad operare decisivi cambiamenti nelle produzioni italiane di inizio Novecento. A partire da tale constatazione, i curatori e le curatrici del saggio preferiscono parlare di "pratiche letterarie in italiano", piuttosto che di "letteratura nazionale italiana", poiché all'interno di una letteratura in lingua, agiscono, dialogano e negoziano modifiche di contenuti e 


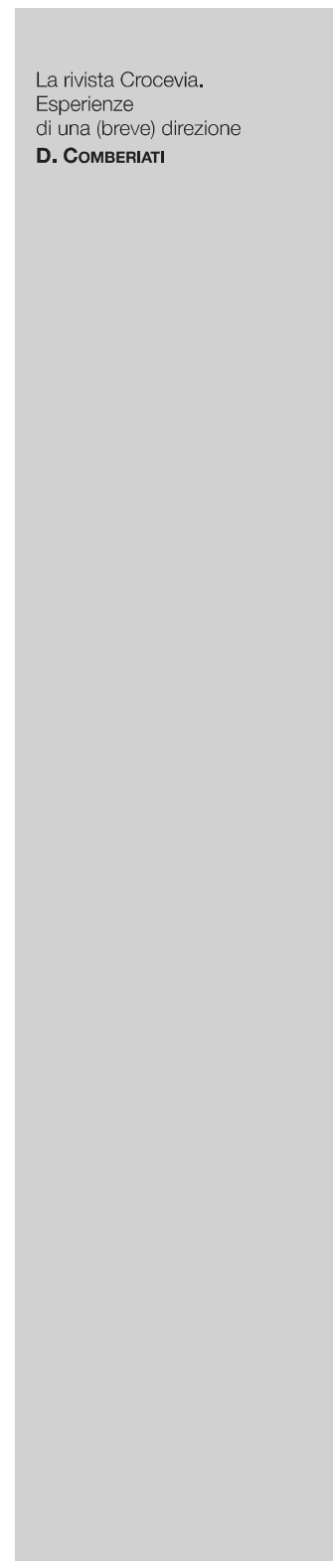

stile anche i testi in traduzione. Come si vede si tratta di un'evoluzione di una contraddizione che già i primi testi migranti lasciavano intravedere e che risponde ancora e sempre alla medesima domanda: ha senso oggi parlare, ancora, di "letteratura nazionale"? Sono i confini geografici e politici che determinano la storia letteraria?

È stato proprio all' inizio delle mie ricerche, mentre stavo ancora ragionando sulla portata e sull'impatto linguistico di queste nuove scritture, che mi sono imbattuto nella casa editrice Besa e nella rivista Crocevia. Conoscevo Besa per il catalogo "albanese", ovvero per aver incluso, fra le sue pubblicazioni, molti scrittori italofoni di origine albanese. Si trattava a tutti gli effetti di una letteratura postcoloniale, ma con sfumature che la rendevano ancora più interessante e complessa. Certamente la relazione coloniale, (semi)coloniale e postcoloniale che si è instaurata negli anni fra Italia e Albania ha contribuito alla conoscenza e alla diffusione dell'italiano dall'altra parte dell'Adriatico. All'interno di un discorso del genere, però, sono diversi gli assi di ricerca che possono essere presi in considerazione: innanzitutto è doverosa una riflessione approfondita su ciò che significa e che ha significato, per entrambe le sponde, il "passaggio" o "attraversamento" del mar Adriatico. Il mare come frontiera, dunque, e il "filtro marino" come specifica condizione dello sguardo del migrante. Ma anche, in una visione più complessa del fenomeno, il mare come oblio, acqua che annega e cancella ricordi ed eventi storici. L'approdo degli albanesi sulle coste italiane negli anni Novanta avrebbe dovuto far riflettere anche su un ulteriore parallelismo storico di cui invece pochi hanno fatto menzione: l'attraversamento di un mare ben più esteso, l'oceano Atlantico, costituiva infatti il percorso dell'esodo migratorio "storico" dall'Italia verso le Americhe nell'Ottocento e nella prima metà del Novecento. Come il Mediterraneo meridionale, nel momento attuale, è diventato il limine valicabile/invalicabile fra due mondi, cosi appariva all'inizio degli anni Novanta il ben più angusto mar Adriatico. Anche alla luce delle odierne politiche italiane e più in generale europee riguardanti i diritti dei migranti e dei richiedenti asilo, non è superfluo riflettere sulle ragioni dell'oblio degli avvenimenti dei primi anni Novanta e sui motivi che hanno portato al ripetersi di alcune dinamiche migratorie e di alcune politiche di accoglienza. Allo stesso modo è inevitabile soffermarsi sulle modalità di analisi e sulle interpretazioni letterarie impiegate dagli scrittori che affrontano questo specifico tema. Gli autori migranti provenienti dall'Albania in che modo descrivono l'attraversamento dell'Adriatico e l'arrivo in Italia? E in che modo la lingua agisce in tale descrizione? Ci troviamo di fronte a uno di quei casi in cui è la letteratura a svolgere la funzione di memoria storica e a costruire una parte dei materiali che formano l'identità di una nazione. Il primo impatto con 


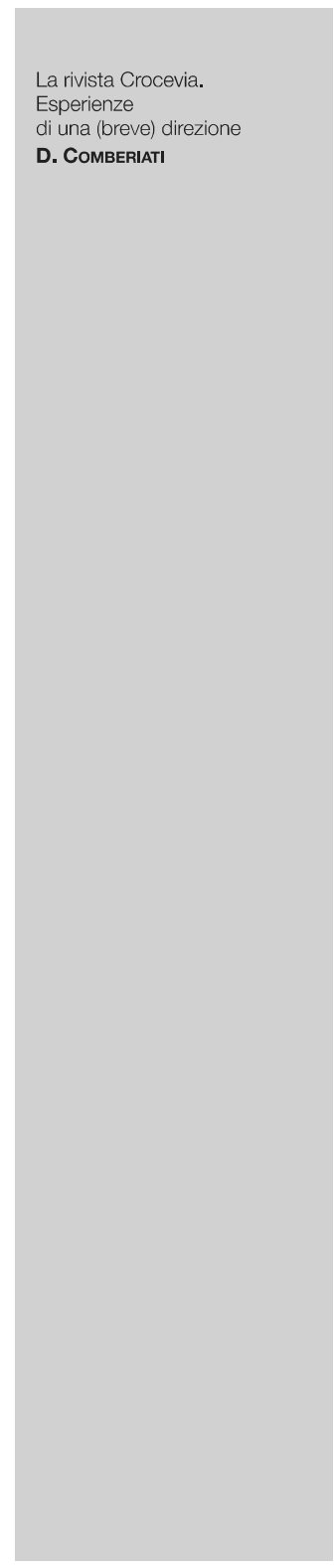

l'Italia, il senso di spaesamento, la determinazione ad apprendere una nuova lingua e una nuova cultura: nei primi scritti degli autori albanesi è possibile rintracciare le relazioni storiche fra i due paesi che hanno caratterizzato gran parte degli anni Novanta.

Essendo ormai impossibile ridurre l'espressione letteraria di una nazione a una lingua, e risultando al tempo stesso inattuale circoscriverla all'interno di confini che vengono frantumati, non solo virtualmente, nel mondo globalizzato, l'analisi di pratiche letterarie alloglotte all'interno di un paese ci permette di riflettere, da una prospettiva diacronica e sul lungo periodo, sui nostri schemi critici, che appaiono talvolta incerti non solo nel rappresentare il mutevole presente, ma anche nel fornire un prospetto globale di un passato storico che appare diverso dalla visione talvolta monolitica dei discorsi ufficiali. In effetti è possibile parlare oggi di "letteratura adriatica" (Scianatico 2011: 25), così come di "letteratura mediterranea", ponendo come punto di riferimento un’identità sovranazionale che accomuna letterati e scrittori più di tante micro-identità locali e che, seguendo le parole della studiosa bulgara Maria Todorova (1997), potrebbe anche fornire una risposta autoctona, nel caso adriatico, alla "creazione" culturale dei Balcani, frutto talvolta di una visione limitata da parte dell'Europa occidentale.

In tal senso la rivista Crocevia mi era apparsa fin dal principio come un esperimento nuovo all' interno dello studio della letteratura migrante, e questo proprio per il suo carattere multiforme; all'epoca, infatti (parlo del 2004-2005) non erano molte le riviste che si occupavano di letteratura della migrazione: vi era stata l'esperienza romana de $I l$ caffe, interessante ma durata pochi anni, e ovviamente già era nata la rivista on-line El-Ghibli, la cui redazione era interamente composta da scrittori e scrittrici migranti e che è tuttora attiva. Attraverso l'operato di Julio Monteiro Martins era attiva anche Sagarana, mentre il resto degli interventi critici o creativi trovava spazio (uno spazio marginale, per la verità) su riviste non specializzate o accademiche, che ovviamente si occupavano anche di altro. La loro presenza su tali riviste era sporadica, limitata ad un numero specifico o ad una tematica particolare. Crocevia invece inglobava tale letteratura (o piuttosto tali pratiche letterarie) all'interno di un contesto più ampio, facendola dialogare con altre letterature migranti in altre lingue, con casi di autori bilingui o plurilingui, con testi tradotti spesso presentati in entrambe le versioni o con opere letterarie provenienti da tradizioni culturali e linguistiche particolari (basca, catalana, aborigena), nelle quali il connubio "nazione/letteratura nazionale" non aveva ragione di esistere. Era questo per me il grande merito e il motivo di interesse nei confronti della rivista, che riduceva e al tempo stesso ampliava a dismisura la portata della letteratura italiana della migrazione: non una semplice conseguenza di un fenomeno sociologi- 


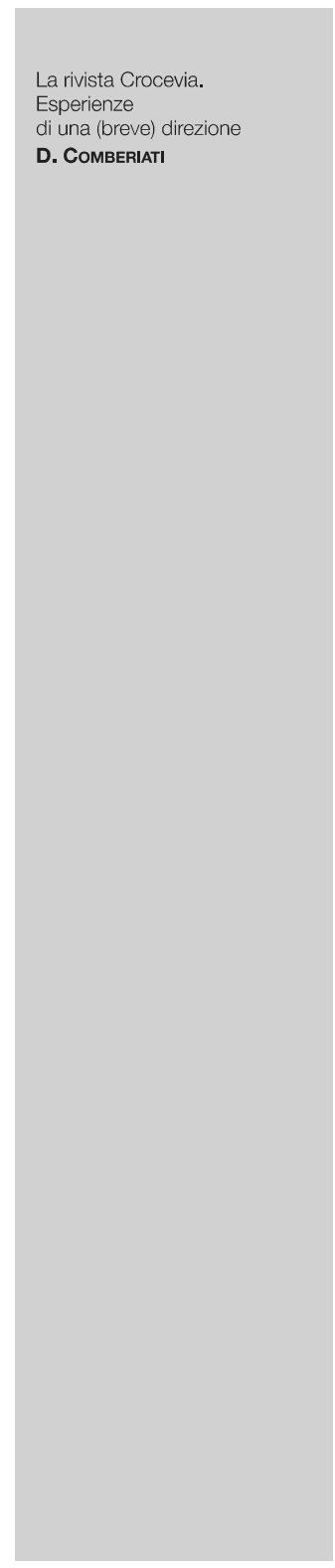

co, ma un grimaldello per guardare diversamente alla storia letteraria mondiale, non solo contemporanea.

Faccio qualche esempio di numeri passati, per dare l'idea della struttura del periodico: nel numero 12 (2008), ad esempio, la parte monografica era consacrata alla nuova letteratura ispano-americana, in un contesto in cui la lingua spagnola (lingua nazionale sui generis, perché coesistente con altri idiomi) si contaminava con altri linguaggi e con percorsi culturali e letterari radicalmente diversi (nel numero erano inclusi autori provenienti da Cuba, Messico, Repubblica Dominicana, El Salvador, Colombia e Cile). La parte "mobile" del numero era invece divisa in tre sezioni: una riguardante i reportage (dal titolo Viaggi di carta \& Carte di viaggio), una la critica letteraria (Appunti in margine) $\mathrm{c}$ infine una comprendente gli scritti creativi (In transito). Da quando ho assunto la direzione (numero doppio 17-18 del 2017) abbiamo cercato di mantenere la struttura, che risultava particolarmente adatta al nostro tipo di discorso sulla letteratura e sulla lingua, e inoltre era riconoscibile dai lettori della rivista, ma ovviamente abbiamo anche apportato alcuni cambiamenti. Innanzitutto abbiamo optato per una scadenza semestrale molto precisa: i numeri escono a settembre e a marzo, per consentire alla rivista di usufruire dei canali di diffusione (librerie, edicole, ma anche festival e fiere letterarie) in modo adeguato. A tale scelta si lega anche l'idea di inserire le presentazioni di Crocevia all'interno di manifestazioni più ampie, per dare da una parte il giusto risalto alla rivista, e dall'altra per evidenziare il taglio specifico del periodico. Le presentazioni alle tre principali Fiere del libro italiane (Milano, Roma e Torino) hanno evidenziato proprio quest'ultimo aspetto: la rivista è stata presentata all'interno di un dibattito sulla letteratura di viaggio (Roma), mettendo in risalto la sezione Vhaggi di carta \& Carte di viaggio, o all'interno di un evento organizzato da "Lingua Madre" a Torino, dando più spazio all'apporto creativo degli scrittori migranti.

Anche dal punto di vista del contenuto abbiamo apportato alcune modifiche: lo spazio monografico è sempre più orientato a presentare quei "limbi" culturali e linguistici che, si tratti di migrazione o plurilinguismo, pongono nuove domande sulla costruzione delle letterature nazionali. Per questo abbiamo presentato nel n. 19 un'antologia di scrittori Rom della Macedonia (utilizzando dunque il concetto di nazione e cultura insieme) e nel n. 20 una selezione di fiabe dei Carpazi, usando come riferimento un elemento geografico, tra l'altro molto vicino a quella che, concretamente, potremmo definire una lunga frontiera in cui diverse voci si incrociano.

La parte saggistica è ora divisa fra le sezioni Radure (saggi veri e propri) e Appunti in margine (riflessioni, recensioni, spunti critici) e cerca di analizzare soprattutto scrittori migranti o postcoloniali, per avere una coerenza 


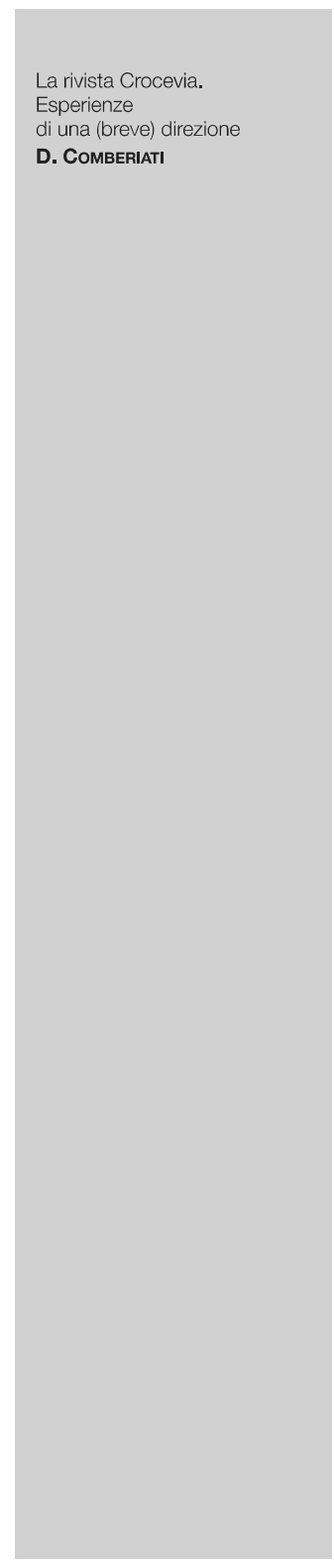

con il resto del numero. Per tale ragione in questi spazi abbiamo presentato finora i lavori dell'italianista egiziana Lamia El Sherif che mette in relazione aspetti della letteratura araba con elementi della letteratura italiana, una riflessione sulla produzione dello scrittore italiano emigrato in Norvegia Luigi Di Ruscio (da parte di Carlo Baghetti), o ancora un articolo sulla fantascienza africana di Oulimata Gueye e la poesia di Afric McGlinchey, che con la sua storia linguistica e letteraria attraversa diversi paesi anglofoni (a cura di Lorenzo Mari). Anche studi italiani su Julio Monteiro Martins (Rosanna Morace) o Rodolfo Wilcock (Sara Di Gianvito) hanno avuto spazio all'interno di tale percorso.

Per quanto riguarda le sezioni creative, abbiamo deciso di "allargare", seguendo il discorso precedentemente accennato, il concetto di letteratura migrante, per proporre non solo testi italofoni, ma tutti quei contributi che ci consentivano di riflettere sulle relazioni fra nazione, lingua e letteratura. Per questo, a nomi già noti della letteratura italiana migrante come Cristina Ali Farah, Nader Ghazvinizadeh, Adrian Bravi e Ron Kubati, si sono aggiunti i contributi di poeti e scrittori internazionalmente riconosciuti come l'haitiano Georges Castera (che ha pubblicato in creolo, in francese e in spagnolo), l'americano-russo Samuel Klonimos o ancora (nel numero 21, in corso d'opera) l'argentino Arturo Carrera, di origine italiana e grande conoscitore e traduttore della letteratura del nostro paese. A partire dal numero 20, inoltre, ha avuto inizio un'importante collaborazione con l'associazione "Lingua Madre", che da diversi anni cura un importante concorso letterario dedicato a donne migranti e italiane e che cura una specifica sezione nella rivista, di volta in volta su una tematica specifica. Le autrici che vi partecipano sviluppano tematiche che stanno a cuore sia alla redazione di Crocevia che all'associazione, creando un ulteriore connubio fra realtà diverse che si occupano di letteratura migrante.

La sezione "Geopoetica" è invece dedicata a testi letterari in traduzione, spesso presentati nella versione originale e in quella tradotta, volti a far ragionare il lettore su realtà linguistiche particolari (il contesto belga, quello catalano, quello occitano) che possano appunto portare ad una riflessione più ampia sul concetto di letteratura mondiale.

Grande spazio è inoltre dedicato alla sezione dei reportage narrativi, perché la rivista, oltre a presentare spunti critici e creativi sulla letteratura migrante, vuole rendere concreto il concetto di "viaggio" in letteratura e dare spazio a tutti quei giovani reporter (italiani e non) che attualmente stanno riscrivendo il canone della letteratura di viaggio. In tal senso, l'apporto di scrittori/viaggiatori (o viaggiatori/scrittori) come Alessandro Pellegatta o Roberto Montella si rivela molto importante, perché grazie ai loro contributi siamo riusciti a far scoprire ai lettori che cosa significa oggi, in un mondo dove il concetto di "sconosciuto" appa- 


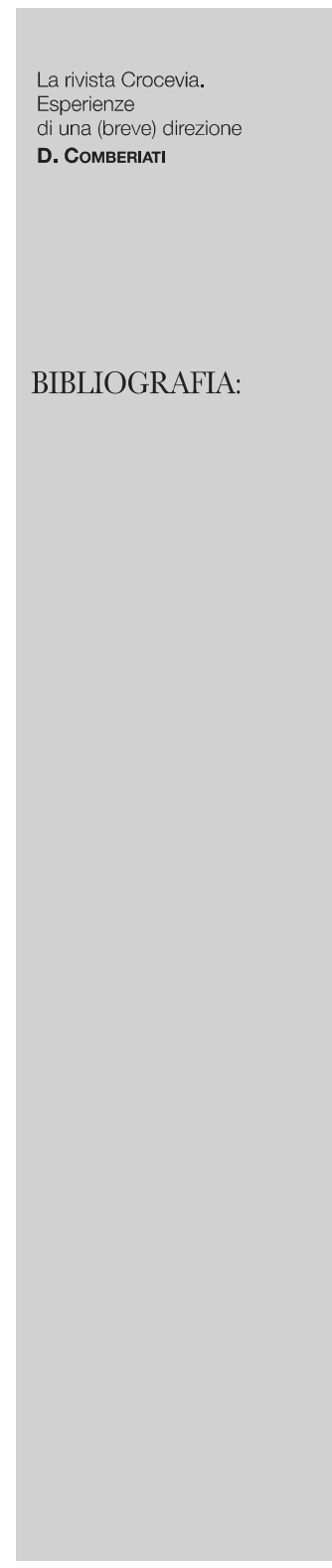

re sempre più lontano, viaggiare per conoscere l'altro (dal punto di vista culturale e linguistico, ma non solo) può ancora essere determinante in vista di una più civile coesistenza fra persone diverse.

$\mathrm{Al}$ di là della sezione specifica, è questo il grande obiettivo di «Crocevia»: creare un ponte, o appunto un incrocio, fra modi diversi di intendere la letteratura e le relazioni complesse e stratificate che si intessono fra scrittore e lingua d'uso, cercando di metterle in relazione con $\mathrm{i}$ cambiamenti degli ultimi decenni e di far riflettere i lettori sull'uso e sulla costruzione del canone delle letterature nazionali.

BALDINI A., BIAGI D., DE LUCIA S., FANTAPPIÈ I., SISTO M 2018. La letteratura tedesca in Italia. Un'introduzione (1900-1920). Maccrata: Quodlibet.

CARPI E. 2007. Letteratura nascente: quale migrazione? in Afriche e Orienti, $\mathrm{n}^{\circ}$ 2, pp. 81-90.

COMBERIATI D. 2008. Scrivere nella lingua dell'altro. La letteratura degli immigrati in Italia (1989-2007). Bruxelles: Peter Lang.

DJEBAR A. 1999. Ces voix qui m’assiègent. Paris: Albin Michel.

GNISCI A. 1998. La letteratura italiana della migrazione. Roma: Lilith.

PARATI G. 1999. Mediterranean Crossroads. Migration Literature in Italy. Madison-London: Fairleigh Dickinson University Press-Associated University Press.

SCIANATICO G. 2011. Per una letteratura adriatica. Lettera Intermazionale, $\mathrm{n}^{\circ}$ 3: 24-26.

SINOPOLI F. 2001. Poetiche della migrazione nella letteratura italiana contemporanea: il discorso autobiografico, pp. 189-213 in Studi (e testi) itatiani. Semestrale del dipartimento di Italianistica e Spellacolo dell'Universilà di Roma La Sapienza, $n^{\circ} 7$.

SINOPOLI F. 2006. Postfazione. Scrivere nella lingua dell'altro, pp. 215-228 in LECOMTE M. (a cura di), Ai confini del verso. Poesia della migrazione in italiano. Firenze: Le Lettere.

TODOROVA M. 1997. Imagining the Balkans. Oxford-New York, Oxford Univ. Press. 